\title{
Diffusion through agar blocks of finite dimensions: a theoretical analysis of three systems of practical significance in microbiology
}

\author{
Arthur L. Koch \\ Author for correspondence: Arthur L. Koch. Tel: +1812855 5036. Fax : +1 8128556705. \\ e-mail: Koch@Indiana.edu
}

Program in Microbiology, Biology Department, Indiana University,

Bloomington,

IN 47405-6801, USA

\begin{abstract}
A number of experimental methods in biology depend on the kinetics of diffusion of a substance through a gel. This paper reviews the diffusion equations, gives the experimental limitations for some useful cases, and presents computer simulations for cases that cannot be treated analytically. While double diffusion is not considered, three single-diffusion situations are treated. (1) Systems for the study of chemotaxis in the gliding bacterium Myxococcus xanthus. Experimental designs used for this in many cases in the literature were inappropriate and mathematical analysis of these is presented. (2) The development of gradient plates. The time necessary for vertical diffusion to become substantially complete and before diffusion in the direction of the original slant has proceeded significantly is calculated. (3) The application to antimicrobial disk susceptibility tests. The basis of the measurement of antibiotic sensitivities with disks containing antimicrobial agents, as routinely used in clinical microbiological and testing laboratories, is analysed and the limitations are assessed and improvements suggested.
\end{abstract}

Keywords: gradient plates, modelling, antibiotic disks, kinetics of flow, chemotaxis

\section{INTRODUCTION}

Analysis of the diffusion of a substance in a solid medium for a variety of geometric situations depends on Fick's diffusion equation for the particular geometric arrangement. Although there is a well-developed theory for diffusion processes, it applies mainly to idealized cases where diffusion takes place in extended infinite environments. Problems and errors arise when these ideal formulations are applied to finite experimental designs.

Many workers have tried to establish stable linear gradients of test substances in agar gels in order to study the hunting behaviour and movements of gliding bacteria, most usually of Myxococcus xanthus. There have been many contradictory claims and interpretations, both for the types of gradients and for the existence of chemotaxis in this organism. In our laboratory, Tieman et al. (1996) devised a way to prepare stable linear gradients by establishing a thin bridge of agar between source and sink blocks of agar. This system was then used to study the movement of single cells or small groups of cells by video microscopy.

The present paper is not primarily concerned with the presence or absence of chemotaxis, but with the basis of the gradients generated by diffusion in different geometrical situations; this includes the justification of the bridge arrangement that we have designed. A major purpose of this paper is to analyse, by computer simulation, the particular geometrical setups used by earlier workers, and the case of the geometry of our bridge apparatus.

The simulation program created to calculate the gradients for this case was then extended to two other quite different cases: the kinetics of diffusion in 'gradient-plate' production, and the kinetics of diffusion of antibiotics from disks placed on the surface of inoculated agar Petri plates. The latter experimental technique is used in clinical laboratories to assess sensitivity to antibiotic agents. Because of the vast number of such assays carried out each year, the 
improvements suggested by these results may have very important practical application.

\section{MATHEMATICAL METHODS}

Diffusion. The mathematics of diffusion as applied to biology has recently been reviewed by Koch (1990) and by Berg (1993). Briefly, to solve a problem in diffusion, either of Fick's laws is applied to every point in the system. Fick's first law can be written for diffusion in one dimension from a space under consideration to an adjoining space in finite form as:

$\Delta q / \Delta t=-A D C / \Delta x$

In this expression, the quantity of substance, $q$, within a finite, but small, space decreases at a rate, $\Delta q / \Delta t$. The loss by outward diffusion is equal to the area, $A$, that separates the space from the adjoining space, times $D$, the diffusion constant, times $C$, the concentration of substance in the compartment under consideration, divided by $\Delta x$, the distance between the spaces. The diffusion constant, $D$, is the proportionality constant; its units are distance squared per unit of time. The value of $D$ depends on the size of the diffusing molecule and is approximately proportional to the reciprocal of the cubic root of the molecular mass.

It could be objected that equation 1 is only part of Fick's first law. First, the amount leaving the source space not only leaves and decreases the concentration $\mathrm{C}$, but augments the concentration in the adjacent space. And second, the reciprocal movement of material from the adjacent space into the space under consideration has not been taken into account. These two points are accommodated in the computer simulation by focussing successively on each space and the movement of material from it to all of its adjacent spaces.

For one-dimensional problems, i.e. those in which the concentration is exactly the same for any values of $y$ and $z$ and only varies for the $x$ dimension, all that need be considered is the net amount flowing from the space in question into its two adjacent spaces. For two- and three-dimensional problems, two or four more additional adjacent spaces in the second and third dimensions must also to be taken into account.

For the analytical calculation of concentration profiles, Fick's second law is frequently appropriate. In three Cartesian dimensions and in partial differential form it is:

$\partial C / \partial t=D\left\{\partial C^{2} / \partial x^{2}+\partial C^{2} / \partial y^{2}+\partial C^{2} / \partial z^{2}\right\}$

With mathematical or computer simulations, the concentration profile at any time is generated by taking into account the appropriate initial and boundary conditions. A standard problem is what is the shape at any time of the concentration distribution of a substance that initially was present as a point source when diffusion occurs indefinitely in one, two or three dimensions. Curves for this are presented in statistical textbooks because the answer is the normal, bell-shaped (Gaussian) curve. For example, bell-shaped curves applying for a different number of dimensions are shown in Berg (1993) and in Koch $(1990,1996)$.

Analytical mathematical treatment of diffusion in a finite environment. When the case of finite slabs is to be considered, one first obtains the mathematical solution for the corresponding case for infinite slabs. Then one takes the portion of the solution beyond the place corresponding to the edge of the actual physical object and inverts this mathematically and adds it to the previous portion of the mathematical solution. One may think of this as putting a reflector at the boundary. It will be necessary to truncate and reflect the infinite solution again and again. Each time one does this, the inverted pieces are added on to construct the final piecemeal solution. Of course, when time becomes infinite the distribution in a finite system with a fixed amount of a diffusing substance must become uniform throughout the system; this fact can be used to confirm that the piecemeal process has been properly carried out. It was also used to test the computer simulations for the cases presented in this paper.

Computer calculation of diffusion in a finite environment. The major purpose of this paper is the study of geometric arrangements that are not simple. The finite difference formulation of equation 1 is the form employed here because simulations of irregular geometries could be treated by applying it to each unit of space for the flow between it and all relevant adjacent spaces no matter how complicated the geometrical situation is.

\section{COMPUTATIONAL CONSIDERATIONS}

Implementation of computer simulation of diffusion in finite environments of arbitrary shape. The simulations reported here of assemblages of finite spaces were executed in BASIC with a Power Macintosh. The time step for the simulation in use has been made so small that a tenfold further reduction does not change the output substantially. The actual program will be sent to persons requesting it. Of course, the programs are readily converted into other languages and adopted to commercial application programs that do simulations. The

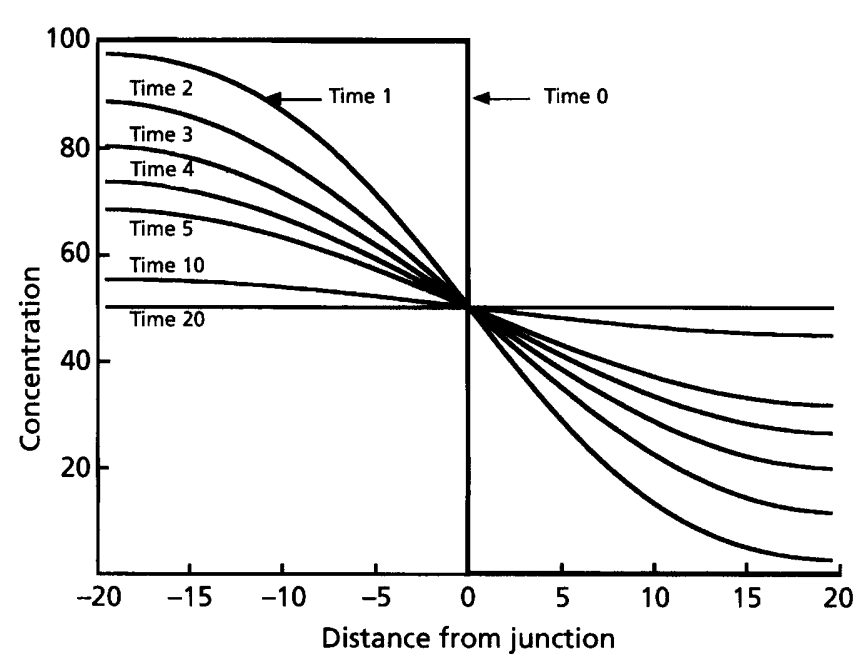

Fig. 1. Diffusion when two identical blocks are placed in contact. This is the geometry used by Dworkin \& Eide (1983). An agar block with a substance of interest is abutted to a block of the the same size without the substance. The diffusion constant is assumed to be the same through both blocks, and the substance diffuses through the joint between the two with the same kinetics as in the blocks. If both blocks were very large, the mathematical theory cited by Koch (1990) and elsewhere would be followed. However, if the blocks are not large and are finite the theory would fail and eventually the concentration would become uniform throughout. While that would take a good deal longer than the experiments of Dworkin \& Eide (1983), calculations for times so long that there is essentially complete equilibration across the system are included. In this case, the gradient becomes shallower, not only because of diffusion, but also because of reflection of the diffusible substance back from the ends of the block. 


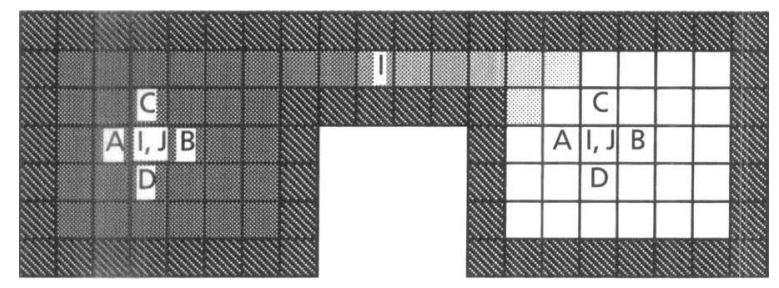

Fig. 2. Setup for simulation of bridge geometry as used by Tieman et al. (1996). The source-sink block and the bridge are encased in a chain of cells (hatched in the diagram) that in the program initially have stored in each the identifying number 200. These are used to allow the program to efficiently feel the edges of the blocks. Initially, the program simulates a substance that is uniformly distributed in the left-hand (nutrient, or $\mathrm{N}$ ) block and none in the right-hand (sink, or S), block. The diagram indicates a later stage when a gradient has been generated in the bridge. In the various parts, the diffusion from a cell or space, $I$ or I,J, is calculated, throughout the bridge and in the directions marked $A, B, C$ and $D$ in both blocks; then by computing the outward flow from every space, summing the values, and then updating the arrays, the course of the diffusion can be followed. This was used not only to measure the kinetics but to establish the stable magnitude of the gradients.

program is simple and many refinements are possible. These refinements would be appropriate for extension to a third dimension and still more complicated geometries. The most important refinement would be to incorporate the RungeKutta procedure, but that was not needed for the illustrative material presented here.

Diffusion between two finite slabs. Fig. 1 shows the simulation for a linear array of 40 spaces of equal sizes. It corresponds to two abutting slabs each of 20 spaces, one slab initially containing a constant concentration of a diffusible substance, and the other containing nothing at time 0 . The concentration profile at this time exhibits a discontinuous step change. For each time cycle the output to and input from the two neighbouring spaces to a particular space is calculated and added. The program treats all spaces in a time cycle. Because the results are stored in a separate set of registers and then this set is used for the next time, the order in which the spaces are treated is immaterial. The important feature is that the program ascertains whether or not there is an adjacent space on either the left or right and transfers accordingly.

For the reservoirs for the bridge geometry, there is a twodimensional problem (see Fig. 2) since there is a possible input from below or above. Similar to the one-dimensional case, the program determines if there are such spaces and then calculates the movement of material between them and the space under consideration.

Simulation for bridge geometries. For the bridge case, there are three regions and the simulation proceeds by setting up six double arrays: two arrays for the bridge, two for the source block, and two for the sink blocks. One array contains the current concentrations and the other contains the change of diffusate concentration in the current time unit. Since this is the heart of the paper the simulation will be examined in a little more detail. Consider just one cell, or space, of the twodimensional array for the nutrient-containing source block, identified by values of $I$ and J of the N2 array (see Fig. 2), the computer would use four equations like:

IF $\mathrm{N} 2(\mathrm{I}-1, \mathrm{~J})=200$ THEN DA $=0$

ELSE DA $=\mathrm{N} 2(\mathrm{I}-1, \mathrm{~J}) \mathrm{N} 2(\mathrm{I}, \mathrm{J})$
There are four equations because the space under consideration can have four adjacent spaces into which the diffusate might flow. DA (and DB, DC and DD of the other three equations for the other three neighbouring cells) is the change in concentration due to the flow into the adjacent region. For convenience and speed in calculation an arbitrary number, which is always 200 , is stored initially in spaces that mark the ends of the blocks, shown as the hatched cells in Fig. 2. The IF/THEN part establishes whether the current position is adjacent to the end of the region. If not, the computer calculates the net change, DA, due to diffusion between the cell at the I,J position and that neighbouring cell. Subsequently, the contribution to the I,Jth cell from the other three neighbours of the I,J cell (DB and DC and DD) would be analogously computed. Then the entire procedure would be repeated for every cell in the $\mathrm{N} 2$ array. After that the changes due to the four adjacent cells to every cell on the sink side (stored in the S2 array) would be calculated in a similar fashion. Then, the total change in the two cells of the $\mathrm{N}$ and $\mathrm{S}$ arrays would be calculated from:

$\mathrm{DN}(\mathrm{I}, \mathrm{J})=\mathrm{DA}+\mathrm{DB}+\mathrm{DC}+\mathrm{DD}$

$\mathrm{DS}(\mathrm{I}, \mathrm{J})=\mathrm{DE}+\mathrm{DF}+\mathrm{DG}+\mathrm{DH}$

This, of course, would then be repeated for all the square arrays in both the N2 and S2 arrays. (For the simulation carried out here, the arrays were 20 by 20 spaces.) A similar process is then carried out for the one-dimensional array containing the spaces in the bridge. Finally the diffusion processes bringing or removing material to or from the cells at the ends of the bridge have to be included. Then the whole cycle is repeated many times, exchanging the data from arrays with suffixes 2 to arrays with suffixes 1 . This switching of arrays is done to eliminate errors introduced when an array is changing while the calculation is going on. At suitable stages the data are output as a file to the graphing program.

Application to other geometries. Since the program tests for the edges of the block (or obstacles in the agar) it does not allow diffusion to move material into (or out of) these places. This approach increases enormously the variety of geometric circumstances that can be readily treated, but it does not generate an analytical solution and it can consume much computer time. However, it has speed and flexibility advantages over some commercial simulation applications. Modification for the case of gradient plates is straightforward. For the radially symmetrical case of antibiotic disks placed on the top of an agar surface, it is only necessary to correct for radial dilution as diffusion spreads the antibiotic from the disk (see below).

\section{RESULTS AND DISCUSSION}

\section{Systems for study of chemotaxis in Myxococcus xanthus}

Diffusion in various finite geometries. A variety of physical setups have been used to study cell movements of various microbes. Most of these earlier techniques (Nelson et al., 1975; Carlile, 1970; Konijn, 1970; Knowles \& Madelin, 1975; Bonner et al., 1966) gave quite rapidly changing non-linear gradients and need not be discussed further here.

Evidence for and against chemotaxis of M.xanthus has been presented. Six early papers (Fluegel, 1963; Ho \& McCurdy, 1979; Jennings, 1961; Lev, 1954; McVittie \& Zahler, 1962; Shimkets et al., 1979) purported to demonstrate chemotaxis. These experimental studies 
were discounted by Dworkin \& Eide (1983) as being insufficient or erroneous. Dworkin \& Eide (1983) then rejected chemotaxis-based mechanisms because of the results of their own experiments. Recently, experimental reports from Zusman's laboratory have claimed to have demonstrated chemotaxis (Shi \& Zusman, 1993; Shi et al., 1993). We (Tieman et al., 1996), on the other hand, rejected the conclusion that $M$. xanthus exhibits directed movements towards a source of nutrition on the basis of experiments with a newly designed experimental bridge setup that produces a linear stable gradient.

Diffusion from one abutting finite slab to another. Dworkin $\&$ Eide (1983) used abutting surfaces of two agar blocks, one initially containing a test substance and the other not. This geometry of two mated blocks has been important in many problems involving the engineering and physics of heat and chemical flows and therefore the solution has often been presented of the concentration distribution at various times after the two blocks (or slabs) have been placed in contact. For the infinite slab case, the well-known solution is simply the integral of the normal curve and is S-shaped. The mathematical solution is given in Dworkin \& Eide (1983) and in Crank (1975, pp. 14 and 15). As emphasized here, these derivations assume that both blocks are infinite in length and of the same fixed width throughout. Except that the two abutting slabs are of finite length, a similar setup is shown in Fig. 1. The figure shows that there is an infinite discontinuity in concentration at time 0 that then becomes progressively more gradual. Although by time 1 the mathematics for the infinite case would still apply because diffusion has not proceeded far enough, at the later times the effects of the finite size of the container greatly alter the idealized infinite case.

The requirement for 'semi-infinite' source and sink block is not restrictive for the geometry and duration of the Dworkin \& Eide (1983) experiments mentioned above. In their case, the dimensions, times and diffusion constants of the substances were such that at the end of the experiments the diffusible substance was neither significantly depleted at the far end of the source block nor had become appreciably greater than zero at the far end of the sink block.

In the simulation, the discontinuity becomes progressively less distinct (see Fig. 1) and by time 20 the distribution throughout the system is substantially uniform. Consequently, the solution does not retain the initial S shape at longer times, and instead throughout the limited space the concentration becomes uniform. Although the gradient remains maximal at the original discontinuity site, it becomes progressively shallower and shallower and finally vanishes. It does not vanish in the case of an infinite slab because the total amount of resource and the amount of vacant space to receive it are both infinite.

The times indicated in Fig. 1 are arbitrary and for actual applications they depend on the value of the diffusion constant, the actual unit of distance, and the dimensions of the blocks. So the actual times could correspond to microseconds to centuries, depending on the circumstances. The indicated times are related to the dimensionless quantity $D t / L^{2}$ as follows: time 1 corresponds to $3300 \mathrm{~s}$ (55 min) if $D=3 \times 10^{6} \mathrm{~cm} \mathrm{~s}^{-2}$ (a diffusion constant of typical of the many nucleotides and cofactors tested by Dworkin \& Eide, 1983) and $L=$ $0.1 \mathrm{~mm}$.

Continuous application of a diffusing substance on the face of a circular slab. Shimkets et al. (1979) devised a special experimental approach, one that involves diffusion in three dimensions. Their experimental arrangement was consistent with the semi-infinite basis of the mathematics used. Their setup involved a continuously replenishing source that maintained the concentration on the surface of the agar constant in a small disk-like region. They recognized the need for large physical dimensions for diffusion to take place, so they designed their experimental setup accordingly and used a 2 litre kitchen bowl. This bowl, filled with agar, to all intents and purposes could be considered as a very large and deep semi-infinite Petri plate; it certainly is large compared to the ordinary $90 \mathrm{~mm}$ diameter Petri plate with a $5 \mathrm{~mm}$ thickness of agar that is used routinely in microbiology laboratories.

Besides the simplification resulting from large size, permitting available equations to be applied, the second key change by Simkets et al. (1979) was to continuously infuse the test substance into a spot at the centre of the agar surface. This allowed a stable gradient to be generated. As the substance diffuses away from the disk area of application, it not only spreads out radially over the surface containing the test organism, but also moves down and spreads throughout the depth of the agar. The concentration at various times and locations can be computed for an infinitely large volume of medium during the transient portion of the diffusion process by the very complex equations presented in Carslaw \& Jaeger $(1959$, p. 215$)$, and at longer times by the equation given in Shimkets et al. (1979). This equation only becomes valid after the gradient has stabilized to a diffusional steady state. These workers chose to develop this system because they knew from the diffusion literature that it would come to a time-independent steady state. However, they did not report any experiments done with it in the paper cited or in their later work. On the other hand, Ho \& McCurdy (1979) reported results using a different, imperfect version of the Shimkets et al. (1979) technique. Ho \& McCurdy (1979) used the usual thickness of agar in the normalsized $90 \mathrm{~mm}$ Petri plate, and instead of a continuous source that was very small, used a disk-shaped application area $7 \mathrm{~mm}$ in diameter; this is clearly not infinitesimal compared with the other dimensions of their system.

Both the Ho \& McCurdy (1979) and the Shimkets et al. (1979) experimental designs require the maintenance of a constant concentration of a test substance in the circular application disk on top of the agar surface. In both cases this was achieved by flushing a solution through a chamber with a disk-shaped permeable 


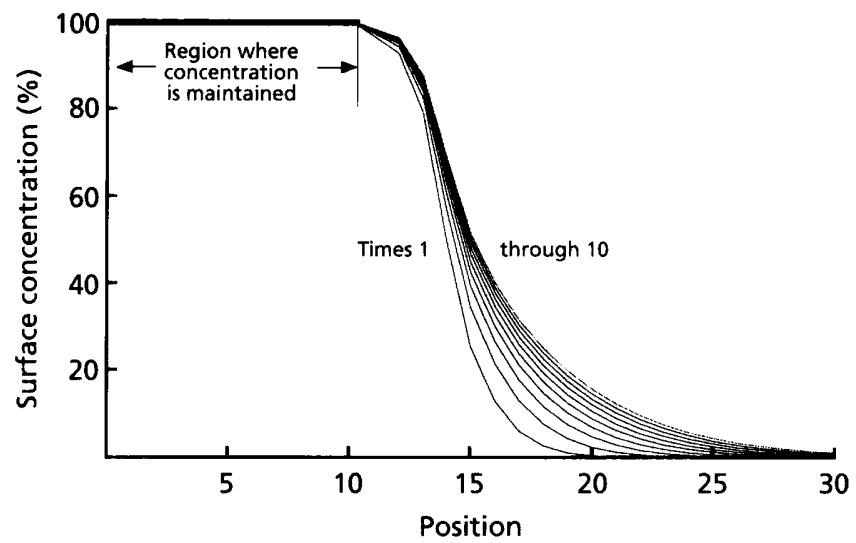

Fig. 3. Cylindrical diffusion in the small system of Ho \& McCurdy (1983). Although this system was modelled on the physical setup proposed by Shimkets et al. (1979), it does not satisfy the idealized criteria, which are the semi-infinite diffusion from a continuous constant point source in cylindrical geometry in three dimensions. Therefore, the assumed mathematical equations do not apply. To see the deviations resulting from these changes, the simulations in this figure and Fig. 4 were performed. This figure presents the surface concentration at various time points. As distinct from the semi-infinite case envisioned by Shimkets et al. (1979), the concentration does not approach a steady state which then remains constant in time and space.

membrane resting on the agar. Consequently, both groups constructed apparatuses that continuously exposed the agar surface in the centre of the agar plate or bowl to a constant concentration of test substance.

The two important sources of deviation from the Shimkets et al. (1979) theory (for a point continuous source on the surface of a deep extended solid) as applied in the Ho \& McCurdy (1979) technique are that the region of application was not even approximately a point source and that the agar layer was not deep. Moreover, the bacteria measured in their studies were located at a radial distance only slightly outside the source disk. The reflection of the diffusing substance from the lower and upper surfaces is important in determining the concentration at the upper margin of the agar, i.e. at the surface where the bacteria were actually located. This reflection effect is particularly important for bacterial cells near to the disk source and would obviate estimation of the local concentration and its gradient by the idealized equations. At large radial distances from the source disk, the diffusion problem approaches that for radial diffusion in a thin agar layer and is different from the solution for the deep agar of the Shimkets et al. (1979) case because the concentration becomes very similar throughout the thickness of the agar.

The simulation shown in Fig. 3 should be an accurate representation of the gradient of concentration in the Ho \& McCurdy (1979) experiment from short to long times. The simulation shows the concentration at the surface of the agar at various times for the case where the radius of the disk is 10 units, the radius of the agar

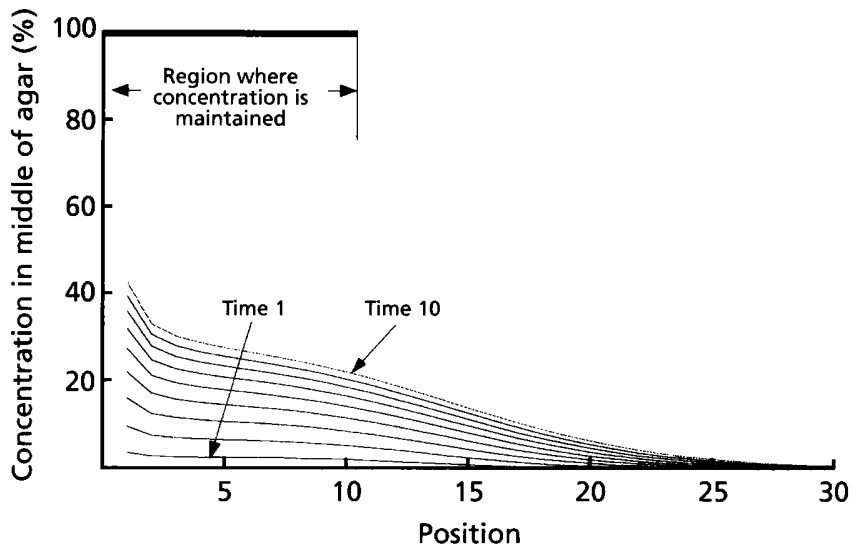

Fig. 4. Diffusion in the depths of the agar for the cylindrical diffusion case. The concentration profile in the middle of the depth of the agar is shown for the setup of Ho \& MCCurdy (1983) described in the text and used in Fig. 3. This too, does not reach a steady state and is very different from the surface concentration profile.

surface is 100 units, and the thickness of the plate is 20 units. This corresponds closely to the dimensions in the Ho \& McCurdy (1979) setup, where 10 units are $7 \mathrm{~mm}$. Fig. 4 shows the distribution in the middle of the agar. Although not shown, the fact that the plate is thin delays the approach to quasi-stability at very early times, and at very long times the reflection from the edge of the Petri plate becomes significant.

The key point of Figs 3 and 4 is that, whereas the Shimkets et al. (1979) system comes to a steady state, the Ho \& McCurdy (1979) system does not. This is because it does not match the well-known mathematical assumptions (point steady-state source in a large deep container) and consequently deviates markedly from the theory. Moreover, the surface concentration gradient (Fig. 3) is steep for only a short distance from the edge of the test substance application area and Fig. 4 shows that the diffusion paths leading to the bottom of the agar and reflecting back to the surface are important.

Another flowing system has been used to study chemotaxis in Dictyostelium discoideum by Fisher et al. (1989), who constructed a system that had two porous circular tubes passing through a thin gel that could be viewed under a microscope. The flow through one tube was essentially the constant source and the other a perpetual sink. This is essentially equivalent to the arrangement of Fig. 5(a). It could serve the same purpose because it is of about the same scale. However, it requires a much more elaborate setup and the geometric situation cannot be analysed with existing analytical equations. It could be with an extensive elaboration of the simulation developed here.

Generation of stable linear gradients with a bridge geometry. The starting point for our development of an apparatus that would have a stable linear gradient was knowledge of the diffusion kinetics from a constant source to a constant sink through a rectangular slab. If 


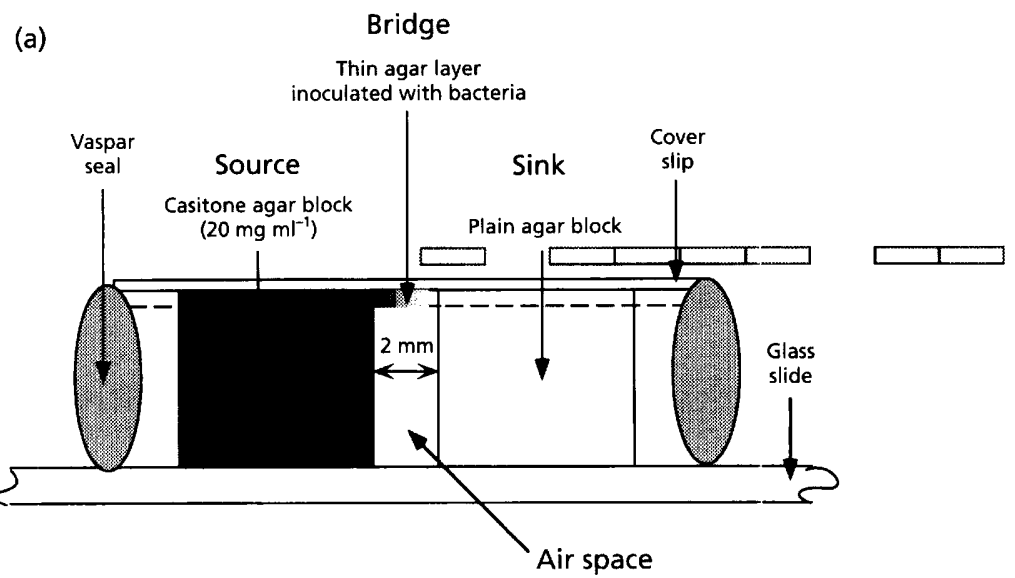

(b)

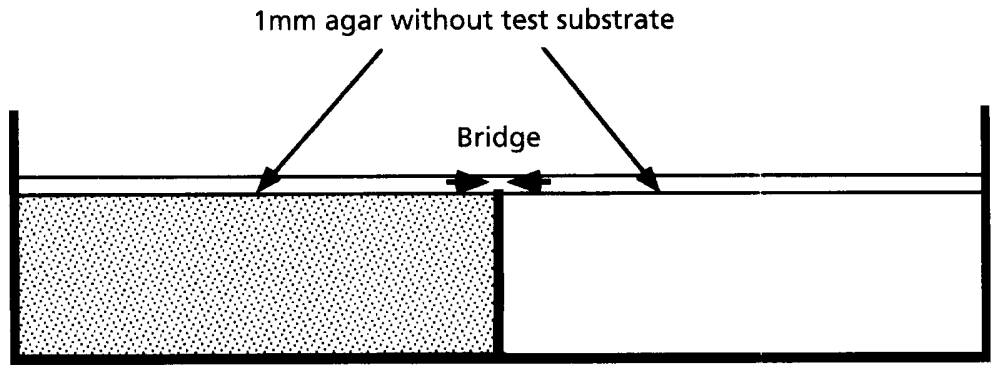

Agar block

with test substance
Agar block

without test substance
Fig. 5. Setups with bridges. (a) The experimental setup used by Tieman et al. (1996). It consists of two agar blocks, the source and sink, bridged by a thin agar layer in which the linear, constant and stable gradient develops. The gradient in the bridge could be approximated by dividing the concentration in the source by the length of the bridge. This would be exactly true if the source and sink were infinitely large and stirring or diffusion within them was fast, or if the bridge was very thin. The fact that this is not exactly so leads to a small deviation estimated through the simulation shown in Fig. 6. (b) Diagram of the system used in Zusman's laboratory (Shi et al., 1993). It is composed of a plastic Petri plate containing a partition or is made by nesting a small Petri plate inside a standard $90 \mathrm{~mm}$ plate. In either case the source agar is connected to the sink agar by pouring a layer to cover the whole plate with a $1 \mathrm{~mm}$ thick layer of agar devoid of the chemical under test. a rectangular block is in contact with a source solution of constant concentration on one side and with a solution kept devoid of the test solution on the other, then a constant linear gradient will develop and afterwards remain stable (see Zigmond, 1977; Lauffenburger \& Zigmond, 1981; Koch, 1990).

That such a system generates a constant linear gradient can be made self-evident from very elementary considerations. Consider that the slab is divided into a series of equal-sized smaller and infinitesimally thin subslabs. The concentration flux across each of these will obey Fick's first law. Since flow out of one is flow into the next, $\Delta q / \Delta t$ will be the same for all the thin subslabs, and $\Delta C / \Delta x$ will be the same for all. Consequently, the concentration will change linearly with distance from the concentration in the nutrient reservoir to zero in the sink reservoir. This result depends on the concentration of source remaining at $C_{0}$ and the concentration at the sink remaining at 0 . This would be strictly true if the source and sink were very large and well mixed. These conditions are approximately but not fully obeyed in the actual experimental setup that Tieman et al. (1996) used.

Bridge geometries can be very useful. Fig. 5 (a) shows the setup of Tieman et al. (1996) and Fig. 5(b), the arrangement of Shi et al. (1993). The former workers placed a source agar block and a sink agar block on a microscope slide and covered both with a cover-slip containing a thin coating of agar that had been inoculated with bacteria. Shi et al. (1993) used plastic Petri plates that contained a partition. They poured agar with additive in one half and additive-free agar in the other. Later they added an approximately $1 \mathrm{~mm}$ layer of additive-free agar to form a bridge between the two chambers. Then they laid down droplets containing either wild-type or mutant $M$. xanthus and observed movement.

The experimental systems developed (Figs $5 \mathrm{a}$ and b) are quite similar to the ideal bridge situation discussed above, and differ from it in only two ways: (i) instead of large and well-mixed solutions, the reservoirs are agar blocks of large, but not infinite, dimensions compared with the bridge; and (ii) the test substances were initially mixed within the congealed agar. Consequently, the test substances move in the blocks by diffusion only and are not kept uniform by mechanical stirring. If the bridges were infinitely thin, then very little material would flow from the source block to the sink block. Under these circumstances, the effect of diffusion within each of the two blocks would be fast enough to serve the same role as would mechanical stirring of the reservoirs; then, diffusion within donor and recipient blocks would be sufficient to maintain the concentration at the edges of the bridge at the original constant values of $C_{0}$ and 0 . Consequently, the generation of a stable steady-state gradient would occur after an initial short time (a few 


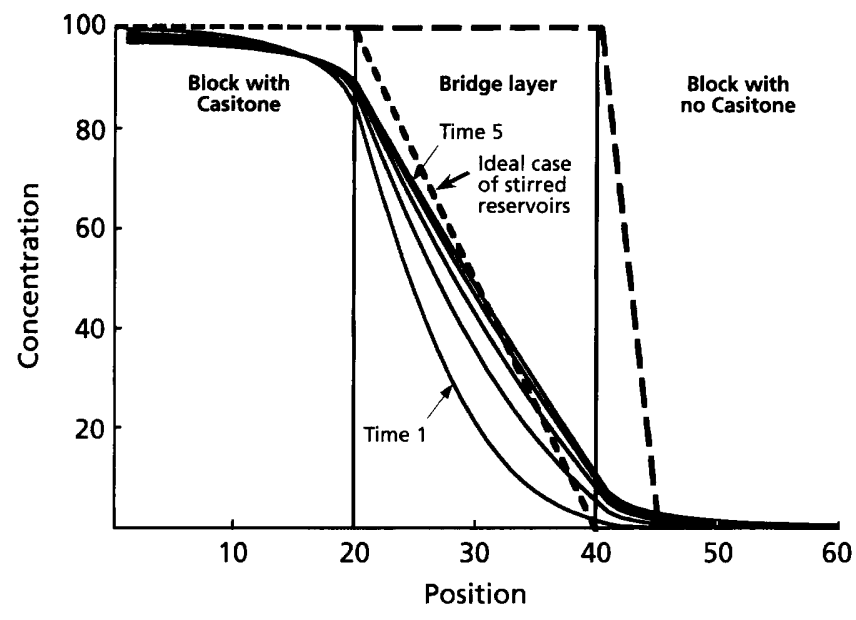

Fig. 6. Bridge diffusion. The gradient in the bridge of the Tieman et al. (1996) apparatus simulated by computer is shown at various times after the assembly of the apparatus. If the source and sink blocks could be replaced by a vessel with continuously stirred contents, then the gradient in the bridge would quickly become linear and the concentration should linearly fall from $100 \%$ to $0 \%$ (short-dashed line). For the nonstirred case shown, the slope becomes linear with about $71 \%$ of the slope that would be observed in the stirred case. This is almost true at any large time when the dimensions of the system are those used in our experimental studies. The final slope is independent of the value of the diffusion constant. The sink side shows the magnitude of the concentration gradients in the experimental design used in Zusman's laboratory. With very much longer times, the gradient in the sink would become shallower and cover a larger region. The line with long dashes indicates the type of (incorrect) extrapolation that Shi et al. (1993) made from their analytical measurements at about $1 \mathrm{~mm}$ from the partition.

hours for the dimensions and materials used). After the linear gradient was generated, its magnitude would be the concentration in the source block divided by the length of the bridge. If the bridge layer was very thin, so that its volume was very small compared with that of the blocks, the gradient would remain stable for a very long time.

If the bridge is not sufficiently thin, then the gradient will be more gradual than this limiting value because the concentration gradient would extend into the two end blocks and the concentration gradient across the bridge itself would be smaller. Near the bridge on the source side the concentration will be less than the original concentration of the reservoir block and on the sink side of the bridge the concentration will be greater than zero. Computer simulation calculations based on Fick's diffusion law for this bridge geometry were carried out. For the dimensions of our agar blocks and the thickness of the bridge it was found that the gradient was about $70 \%$ of the value that would be observed for an infinitely thin bridge (see Fig. 6). This value is consistent with the control observations of Tieman et al. (1996) and applies very nearly both to their setup and to the several variations used in Zusman's laboratory. The decrease in slope is dependent on the ratio of the thickness of the bridge relative to the thickness of the blocks of agar. For the calculations presented in Fig. 6 the ratio of thicknesses of bridge to block is $1: 20$; this is exactly the condition we used.

In the experiments from Zusman's laboratory the organisms were tested on the sink side of the partition. It can be seen in Fig. 6 that the maximum concentration on the sink side portion of the system is quite small. Moreover, the gradient is not constant and it is not steep. Thus, it can be concluded that the gradients produced on the sink side of the bridge are very shallow and do not stabilize with the experimental arrangement used by Shi et al. (1993).

There are difficulties with experimental estimation of the gradient on the sink side of the bridge. The Shi et al. (1993) case is, actually, much more complicated because the thin bridge of agar over the plastic barrier is only at one edge of the thicker agar of the sink compartment where the bacterial movements were actually studied. Thus, the three-dimensional diffusion problem relevant to their experimental design is actually a flow disseminating through the entire sink block after passage across the bridge. As mentioned, this diffusion problem cannot be solved analytically. This is because the test substance gradually moves from an ever-increasing depletion zone on the supply side and flows into the initially substance-free block. This becomes gradually filled with diffusate near the bridge as material moves into the sink and occupies an ever-expanding zone that includes not only the surface, but also the subsurface depth of the agar. Evidently the measurements that the authors carried out to test the gradient were appropriate, but they could not measure the concentration at the very edge of the bridge and at the agar surface. They (incorrectly) assumed that it was equal to that in the original source block on the other side of the partition. The nearest measurement actually made was $1 \mathrm{~mm}$ away from the barrier (see Fig. 1 of Shi et al., 1993). There the concentration was about $10 \%$ of that on the original supply side. These measurements were experimentally difficult because it is the surface concentration, where the bacteria were located, that is relevant. However, if they sampled throughout the depth of the agar then the results would not correspond to the surface concentration because the values would be an average over all depths and would not be a constant proportion relative to the surface concentration, particularly close to the outfall from the bridge. Comparison of the result of the present analysis shown in Fig. 6 with Fig. 1 of Shi et al. (1993) leads to the conclusion that their measurements were probably correct, but they then made an incorrect extrapolation to the surface concentration at the sink end of the barrier. Their extrapolation is shown by the long dashes in Fig. 6.

\section{Kinetics of establishment of stable gradients in gradient plates}

One-dimensional gradient plates are used in microbiology to study many phenomena and could be used 


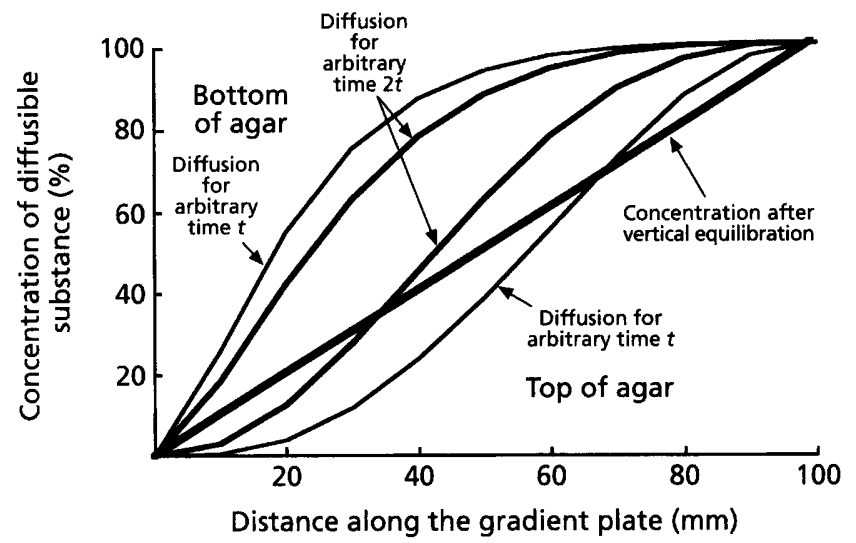

Fig. 7. Approach to equilibrium during preparation of a gradient plate. The figure shows the process of vertical equilibration during gradient plate manufacture. It is assumed that the substance of interest is incorporated in the lower layer. The thickest line shows the concentration after diffusion to vertical equilibrium in the absence of lateral diffusion. The thinnest lines show the concentration after an arbitrary period of time; the lines of intermediate thickness correspond to diffusion periods twice as long.

profitably to study many more aspects of microbial physiology. The simulations of the stability of the gradients presented here may encourage workers to utilize this technique. These plates are made by placing the (usually, rectangular) Petri plate on an inclined surface and pouring one aliquot of agar with some additive to be tested. Once the agar hardens, the plate is placed on a level surface and the same volume of additive-free agar is poured on top. If the slope and volumes are appropriate, after some time has been allowed (usually overnight in the cold) for the additive to diffuse vertically essentially to equilibrium, the plate is inoculated and incubated. The computer simulation developed for Fig. 1 could be used for the process of gradient-plate diffusion at one point of the plate. This is where the top and bottom layers have the same thickness. Although at every point on the interface between the layers at the time the second layer congeals there is an infinite discontinuity in concentration, except at the centre of the plate the diffusion path is longer in one layer than the other. Consequently, in the middle of the plate the equilibration is faster. Near either end, one layer is thicker than the other and diffusion takes longer to fully equilibrate the concentration throughout the gel. These diffusion processes can be simulated with the same program as used for Fig. 1 by increasing the number of filled compartments on one side and decreasing them on the other. After time intervals that are large enough to allow the diffusion in the vertical direction to nearly approach equilibration in the middle of the plate, near either end of the gradient the equilibration is far from as complete (Fig. 7). It turns out, however, that for molecules that are small $(<1 \mathrm{kDa})$, overnight storage of the agar plate is sufficient for substantially complete equilibration even near the ends (corresponding to the straight line in Fig.
7). Of course, the plates cannot be stored indefinitely because then equilibration across the width of the plate from one end to the other would dissipate the lateral gradient. Therefore, the user of the gradient plate needs to wait long enough so that the gradient becomes dissipated in the up/down direction through the thickness in the Petri plate, but not so long that the gradient from the high end to the low end becomes dissipated. For $1 \mathrm{kDa}$ sized molecules, it takes months for the diffusion of an initially linear gradient to become homogeneous across the length of the $100 \mathrm{~mm}$ plate. However, for test molecules that are reasonably small, using the plates the day after preparation seems to be a good compromise.

Double diffusion gradients. Double diffusion gradient plates have been very useful in analysing bacterial growth processes because various combinations of physiological parameters correspond to particular points on the double gradient. A key reference that will lead back to other studies is Thomas \& Wimpenny (1996). The only relevant point from the present study to this field depends on the fact that the gradient of diffusion in one dimension is independent of the diffusion in the second. Consequently, times to equilibrate in the two dimensions can be calculated as described in the preceding section. Since the diffusion constants for the two substances are known it can be calculated whether sufficiently stable gradients are present in both dimensions.

\section{Antibiotic disk assays}

The use of diffusion through agar of antibiotics has long been an important tool for identifying sensitivities and resistances of bacteria, and is used in the laboratories of producers of antibiotics (Cooper, 1962, 1972). Various geometries for the diffusion have been used, but the development of paper disks impregnated with antibiotics turned out to be convenient (Hewitt, 1977; Hewitt \& Vincent, 1989). It came into common usage in the 1960s (see Murray et al., 1995; Mahon \& Manuselis, 1995), and since the 1970s the official usage and procedures in the USA have been defined by the National Committee for Clinical Laboratory Standards (1993).

In the clinical laboratory, paper disks impregnated with antibiotics are dispensed from a device that places a number of disks in a circular arrangement around a plate previously inoculated over its entire surface with the organism to be tested. The various disk cartridges installed in the dispenser have a variety of different antibiotics chosen to be relevant to the type of organism being tested. Primarily this procedure is used to test an organism recovered from a patient to establish the antibiotics to which it is sensitive. The plate is incubated and examined after a prescribed period. With an organism sensitive to the antibiotic there is a circular zone of inhibition around the disk. When a plate has been inoculated with resistant organisms, the growth of cells is constant up to the edge of the disk. The diameters of the zones of inhibition are measured and compared 


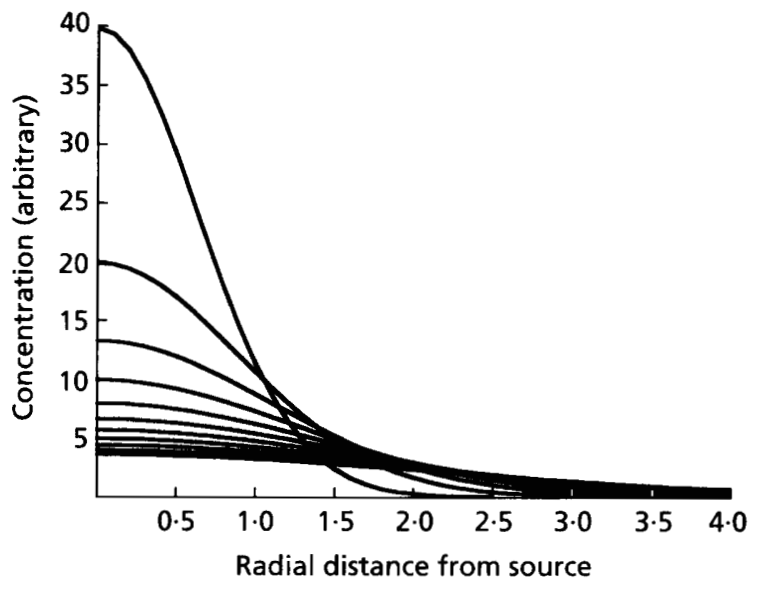

Fig. 8. Diffusion from a point source in two dimensions. Concentration profiles are shown for cylindrical diffusion from an infinitesimal source. Each line represents data for a different time point. With increasing time the peak concentration decreases and the profile becomes broader. At the initial time, time 0 , the profile would be a vertical line of infinite height

with tables for different organisms and different antibiotics to establish the sensitivity or resistance of the isolate in question.

Idealization of the actual working conditions. First let us consider the idealized case and then the application of the computer modelling for the conditions actually used in practice. Ideally, the disk is very small in radius, the agar layer is very thin, the bacteria do not alter the antibiotic, and the flow of the antibiotic through the agar follows the radial law for two-dimensional cylindrical diffusion. This law is:

$C=\{A / 4 \pi D t d\} \exp \left(-r^{2} / 4 D t\right)$

where $C$ is the concentration of the antibiotic, $A$ is the amount of antibiotic in the disk, $D$ is the diffusion constant in agar, and $d$ is the depth of the agar plate. As a function of the distance, $r$, from the origin, the concentration profile is the Gaussian, bell-shaped, normal curve at any time, $t$, but the proportions of the bellshaped curve change with time (Fig. 8).

Evidently at any point on the agar surface, the bacteria will grow until the antibiotic concentration becomes large enough to be inhibitory. If this occurs early enough, then no growth may be observable (except for that of rare pre-existing mutants). If the critical concentration is only achieved late, then the cells will have multiplied enough and the region may remain turbid (unless the antibiotic causes bacterial lysis). For the idealized case, if the amount applied, $A$, the critical concentration, $C_{c}$, and the critical time, $t_{c}$, were known, then the radius that gives an intermediate opacity between the detectable and undetectable levels of growth, $r_{\mathrm{c}}$, could be calculated from a rearrangement of equation 5 :

$r_{\mathrm{c}}^{2}=-4 D t_{\mathrm{c}} \ln \left(C_{\mathrm{c}} 4 \pi D t_{\mathrm{c}} d / A\right)$

Calculations from this formula are shown in Fig. 9 for

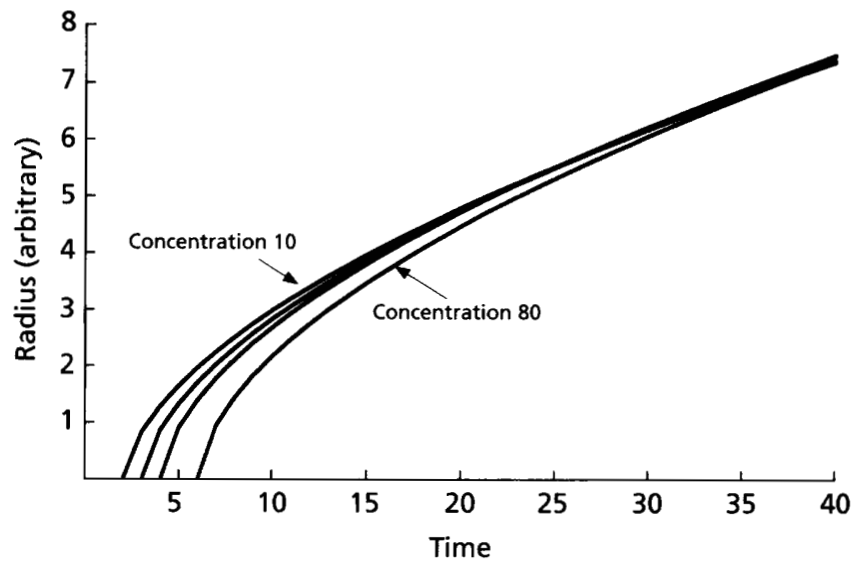

Fig. 9. Radius/time combinations corresponding to achieving the same critical concentration of antibiotic. The calculations are for the idealized two-dimensional cylindrical diffusion and the assumption that the concentration rises at the relevant parts of the agar of the plate until a critical concentration $C_{c}$ is achieved. The lines represent critical concentrations of 10,20 , 40 and 80 (arbitrary units).

twofold increments of the critical concentration. It can be seen that there is relatively little change if all conditions remain the same except that one organism is less sensitive than another organism. This applies unless conditions were chosen so that only very small zones of inhibition are generated. The practical point is that if the disks could be made much smaller (so that smaller zones could be accurately measured) or if conditions were such that the bacteria grew more slowly, then the ability to distinguish different degrees of antibiotic sensitivity would be greater.

For most clinical applications, a range of antibiotics are applied in the disks to the plate and after incubation the diameters of the zones of inhibition are measured. Manufacturers of the disks choose levels of antibiotics so that an easily detectable zone of inhibition can be observed when sensitive bacteria are spread on the plate. Consequently, the concentration of the drug chosen to be applied on the disks depends on its efficacy, and also on its molecular size, since a large molecule will diffuse more slowly than a small one.

Factors affecting size of the zone of inhibition. There are many factors that determine the size of the inhibition zone (see the various articles, books and manuals cited above). Evidently, a larger amount of antibiotic applied would lead to a larger zone of inhibition. It is also expected that lowering the incubation temperature below the growth optimum would slow diffusion slightly, but would slow growth more. Consequently, if the technician incubated the plate longer at a lower temperature, to compensate for slowed growth, then the measured zones would be larger. However, if diffusion was very rapid compared with growth, then the antibiotic may have diffused too far and prevented growth over a very large zone or over the entire plate. Possibly, 
the antibiotic may have become so diluted that growth occurs over the entire plate and no zones of inhibition develop.

In reality the edges of zones are often quite fuzzy and it is of practical interest to ask how they could be made sharper and more distinct. In some cases the biology of the organisms helps; for example, penicillin acts weakly on almost stationary-phase colonies of some strains and they continue to grow slowly, thus enhancing contrast. From Figs 8 and 9 it can be concluded that smaller zones will have sharper margins.

Determination of the critical concentration and time. Although in clinical practice just the sensitivity of a strain to an antibiotic is obtained from the disk test, more could be learned. Both $C_{c}$ and $t_{\mathrm{c}}$ could be determined if, instead of a variety of different types of antibiotics, all the cartridges in the disk dispenser contained a single antibiotic in a range of amounts. These desired parameters could be obtained by rearranging equation 5 ; this gives:

$\ln (C)=\ln (A / 4 \pi D t d) r^{2} / 4 D t$

Adding a subscript ( $\mathrm{c}$ for critical) pertaining to the edge of the zone of inhibition, we obtain:

$\ln \left(C_{\mathrm{c}}\right)=\ln \left(A / 4 \pi D t_{\mathrm{c}} d\right)-r_{\mathrm{c}}^{2} / 4 D t_{\mathrm{c}}$

This is another form of equation 6. Of course, $\ln \left(C_{\mathrm{c}}\right)$ and $\ln (1 / 4 \pi D d)$ are the same no matter what the amount of antibiotic. Consequently, the plot of $\ln (A)$ versus $r_{\mathrm{c}}^{2}$ should yield a straight line with the slope $\left(1 / 4 D t_{\mathrm{c}}\right)$ and intercept set by the equation

$\ln \left(A_{0}\right)=\ln \left(C_{\mathrm{c}}\right)+\ln \left(4 \pi D t_{\mathrm{c}} d\right)$

where $A_{0}$ is the extrapolated amount that gives no zone of inhibition, i.e. $r_{\mathrm{c}}=0$. If the diffusion constant, $D$, for the antibiotic is known and the depth of the agar, $d$, is known or measured then $C_{\mathrm{c}}$ and $t_{\mathrm{c}}$ can be calculated from the slope and intercept. This strategy has been used in the past in experimental studies, but is not part of current clinical practice. However, I suggest that it might be reinstituted and have an important use in the treatment particularly of chronic infections.

From the value of $t_{\mathrm{c}}$, estimated in this way, clinically useful results might result of the time that an individual cell needed to grow in order to become a sufficiently large microcolony to be effectively antibiotic resistant. For many antibiotic/organism combinations, the drug, acts only on growing cells. Thus, when the plate is started and the organisms are spread on its surface as well-separated, growing cells, then the drug at adequate concentrations can inhibit growth. However, if the arrival of a sufficient concentration of the antibiotic is delayed, then each cell may have grown into a micro-clone that now grows more slowly because it has depleted the local environment of nutrients, secreted toxic products, induced a resistance mechanism, or just: become overgrown within the microcolonies. All these points are clinically relevant. Estimation of $t_{\mathrm{c}}$ can determine the time for the microcolony to reach this state. Such an approach has been used in the past for antibiotics (see Cooper, 1962, 1972), and also to define

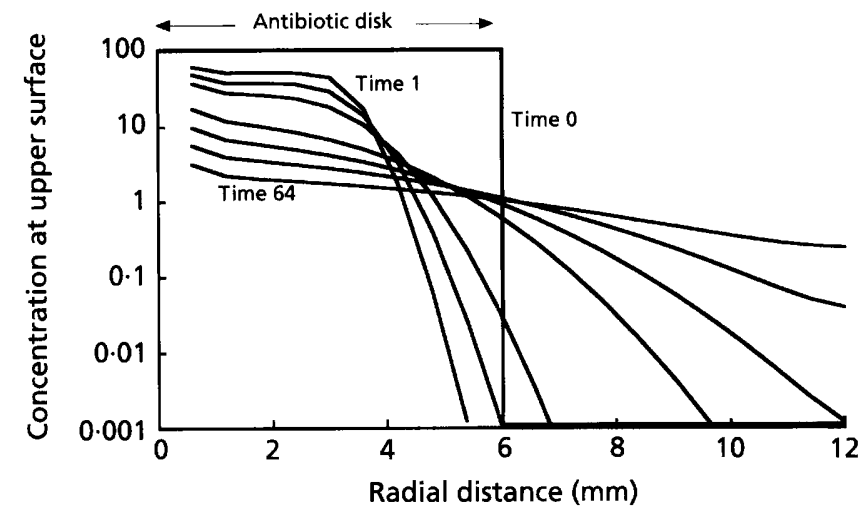

Fig. 10. Concentration as a function of distance from the centre of the disk on the surface of on agar plate. The simulation has been scaled to be directly applicable to the standard way in which antibiotic disk assays are done. The unlabelled lines correspond to successively twofold larger times. Notice that the ordinate is semilogarithmic. It can be seen that the gradient away from the disk is never very great; this gradual concentration change results in blurred edges of the zones of inhibition, making them difficult to measure.

the time that a single bacterium would take to yield a microcolony that became resistant to phage T4 infection (Koch, 1959). This could be accomplished by using T4 coliphage infection of Escherichia coli B under conditions where the virus and the bacteria were mixed together and spread all over the plate. This worked because tryptophan, the necessary cofactor for adsorption for the virus, was applied at different levels to holes cut through the agar layer at various locations on the plate. In this case it was found that when an individual E. coli cell had grown to 50 individuals, the growth of T4 was blocked.

Simulation for agar slabs that are not infinitely thin and disks that are not infinitely small. The above sections are based on the ideal way of carrying out the antibiotic disk assay. They depend on the analytical equations that can be manipulated and relations concerning the concentration versus position and time. The actual problems posed by the current technologies are harder and require computer simulation; in this section I describe the use of the computer to simulate the diffusion of a substance from a disk on the top of the agar plate into the agar and effectively being reflected from the bottom and top surfaces. The substance also diffuses radially until the walls at the sides of the plate are contacted and then is reflected from these surfaces as well. The latter is sufficiently unimportant that it will be omitted below. However, the reflection from the upper and lower surface of the agar is a significant factor. The simulations calculated the distribution of the concentrations as a function of time, depth and radial distance for the typical case of $6 \mathrm{~mm}$ disks and 3-4 mm deep agar. Fig. 10 shows only the concentrations on the top of the agar where the bacteria are growing; Fig. 11 shows the concentration on the bottom to demonstrate the importance of the vertical part of the diffusion. It can be 


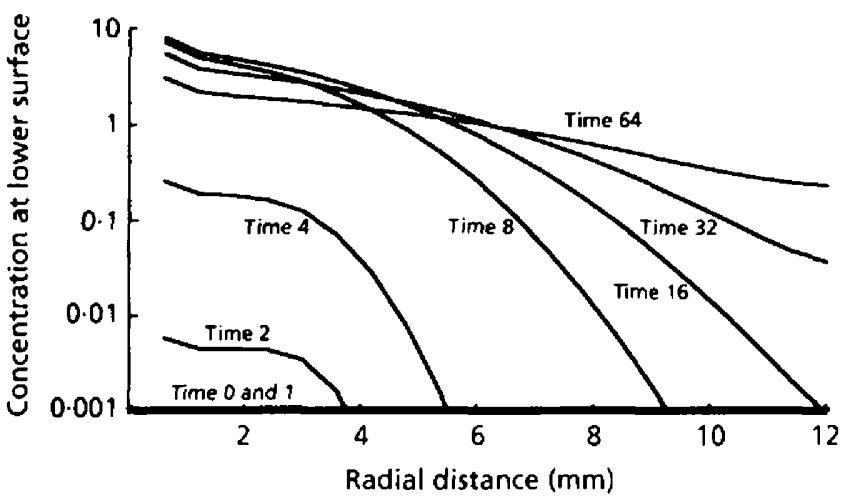

Fig. 11. Concentration on the bottom surface as a function of distance from the centre of the disk. This figure is for the same simulation as that of Fig. 10. The figure shows that with the standard method for antibiotic testing only after a long time does the concentration on the top and the bottom approach equality.

seen that by time 64 the drug has pretty well equilibrated in the vertical direction and then the diffusion follows the equation for cylindrical diffusion (i.e. equation 5).

\section{Conclusions}

The echnique of diffusion of substance through solidified media could be utilized much more intensively in the microbiological laboratory to answer fundamental and practical questions. The primary purpose of this study was to investigate the construction of stable linear gradients for studies of the behaviour and motion of myxobacteria. This led to application to two areas of mort general interest. The practical production of diffusion gradient plates in terms of required diffusion times before utilization is defined. Of major interest is the analysis of the diffusion mechanics of the antibiotic disk assay as used in clinical laboratories. The assay procudure has been analysed in depth and ways to improve and extend it have been suggested.

In the future, antibiotic sensitivity tests could be reengincered so that instead of disks, porous necdles with the substance to be tested would be driven through the thickness of the agar. Since the initially antibioticcontaning region would be narrow, the simple analytical theory presented, only requiring the use of a calculator, would work directly; and because the needles would penetrate the agar, the computations of the profiles of concentration through the depths of the agar would become unnecessary. Finally, because the working zones of inhibition could be smaller, many more kinds of antibiotics or concentrations of the antibiotics could be used on a single plate.

The computer simulation programs are available from the author and can be used for other problems such as diffusion of materials through any heterogeneous cnvironment. It would be possible to study diffusion through irregular spaces that could contain arbitrary obstacles.

\section{ACKNOWLEDGEMENTS}

I wish to thank Martin Dworkin, George Hegeman, Rick Mower and David White for helpful discussions. This project was supported in whole by BRSG (irant RR703126 by the Biomedical Research Support Grant Program, Division of Research Resources, National Institutes of Health awarded to David White and Arthur Koch.

\section{REFERENCES}

Berg, H. (1993). Random $W_{a}$ alks in Biology, 2nd dn. Princeton, NJ: Princiton University Press.

Bonner, J. T., Kelso, A. P. \& Gillmore, R. G. (1966). A new approach to the problem of aggregation in the cellular slime moulds. Biol Bull 130, 28-42.

Carlile, M. J. (1970). Nutricion and chemotaxis in the myxomycete Physaram polycephalum: the cffect of carbohydrates on the plasmodium. I Gen Microbiol 63, 221-226.

Carslaw, H. S. \& Jaeger, J. C. (1959). Conduction of lieat in Solids, 2nd edn. London: Oxford University Press.

Crank, J. (1975). The Mathematics of Diffusjon. Oxford: Clarcndon Press.

Cooper, K. E. (1962). The theory of antibiotic inhibition zones. In Analyicial Microbiology, pp. 1-86. Fdited by F. Kavanagh. New York: Academic Press.

Cooper, K. E. (1972). The theory of antibiotic inhibition zones. In Analytical Microbiology, vol. 2, pp. 13-30. Fdited by F. Kavanagh. New York: Academic Press.

Dworkin, M. \& Eide, D. (1983). Myxococitus xunthus does not respond chemotactically to moderate concentration gradients. J Bacteriol 154, 437-442.

Fisher, P. R., Markl, R. \& Gerisch, G. (1989). Quantitative analysis of cell motility and chemotaxis in Dictyostelium discoiderm by using an image processing system and a novel chemotaxis chamber providing seable chemical gradients. I Cell Biol 108, 9.7.-984.

Fluegel, W. (1963). Fruiting chemotaxis in Myxococcus xanthus. Proc Minn Acad Sit 32, 120-123.

Hewitt, W. (1977). Microbiological Assay: an Intruduction to Quantitatil ' Principles. Ncw York: Academic Press.

Hewitt, W. \& Vincent, S. (1989). Theory and Appliciation of Microbiolugical Assay. New York: Academic Press.

Ho, J. \& McCurdy, H. D. (1979). Demonstration of positive chemotaxis by cyclic GMP and 5'-AMP in Myxococcus xanthus by means of a simple appararus for generating practically stable concentration gradients. Can J Microbiol 25, 1214-1218.

Jennings, J. (1961). Asscciation of a steroid and a pigment with a diffusble fruting factor in Myxococas tirescens. Nature 190. 190.

Knowles, D. \& Madelin, M. F. (1975). Chemotactic and other responses of the plasmodia of Bulhamia utricularis to an extract of Stereum hirsuttem and to certain other substances. I (ien Microbiol 89, 23.5-244

Koch, A. L (1959). The dynamics of coliphage plaque formation. I. Macroplayue experiments. Virology 8, 273-292.

Koch, A. L. (1990). Diffusion: the crucial process in many stages of the hiology of bacceria. Ade Microb Ficol 11, 37-70.

Koch, A. L. (1995). Bacteral Grouth and Form. New York: Chapman \& Hall.

Koch, A. L. (1996). What size should a bacterium be? A question of scale. Annu Rev Microbiol 50, 317-318. 
Konijn, T. M. (1970). Microbiological assay of cyclic $3^{\prime}, 5^{\prime}$-AMP. Experientia 26, 367-369.

Lauffenburger, D. A. \& Zigmond, S. H. (1981). Chemotactic factor concentration gradients in chemotaxis assay systems. J Immunol Methods 40, 45-60.

Lev, M. (1954). Demonstration of a diffusible fruiting factor in myxobacteria. Nature 173, 501.

Mahon, C. R. \& Manuselis, G., Jr (1995). Diagnostic Microbiology. Philadelphia: W. B. Saunders.

McVittie, A. \& Zahler, S. A. (1962). Chemotaxis in Myxococcus. Nature 194, 129-130.

Murray, P. R., Baron, E. J., Pfaller, M. A., Tenover, F. C. \& Yolken, R. H. (1995). Manual of Clinical Microbiology, 6th edn, pp. 1337-1340. Washington, DC: American Society for Microbiology.

National Committee for Clinical Laboratory Standards (1993).

Performance standards for antimicrobial disk susceptibility tests. Approved Standard M2A5. Villanova, PA: National Committee for Clinical Laboratory Standards.

Nelson, R. D., Quie, P. G. \& Simmons, R. L. (1975). Chemotaxis under agarose, a new simple method for measuring chemotaxis and spontaneous migration of human polymorphonuclear leucocytes and monocytes. J Immunol 115, 1650-1656.

Shi, W. \& Zusman, D. R. (1993). The two motility systems of Myxococcus xanthus show different selective advantages on various surfaces. Proc Natl Acad Sci USA 90, 3378-3382.

Shi, W. \& Zusman, D. R. (1994). Sensory adaptation during negative chemotaxis in Myxococcus xanthus. J Bacteriol 175, $1517-1520$

Shi, W., Köhler, T. \& Zusman, D. R. (1993). Chemotaxis plays a role in the social behaviour of Myxococcus xanthus. Mol Microbiol 9, 601-611.

Shi, W., Köhler, T. \& Zusman, D. R. (1994). Isolation and phenotypic characterization of Myxococcus xanthus mutants which are defective in sensing negative stimuli. J Bacteriol 176, 696-701.

Shimkets, L. J., Dworkin, M. \& Keller, K. H. (1979). A method for establishing stable concentration gradients in agar suitable for studying chemotaxis on a solid surface. Can J Microbiol 25, 1460-1467.

Thomas, L. V. \& Wimpenny, J. W. T. (1996). Investigation of the effect of combined variations in temperature, $\mathrm{pH}$ and $\mathrm{NaCl}$ concentration on nisin inhibition of Listeria monocytogenes and Staphylococcus aureus. Appl Environ Microbiol 62, 2006-2012.

Tieman, S., Koch, A. L. \& White, D. (1996). Gliding motility in slide cultures of Myxococcus xanthus in stable and steep chemical gradients. J Bacteriol 178, 3480-3485.

Zigmond, S. H. (1977). Ability of polymorphonuclear leukocytes to orient in gradients of chemotactic factors. J Cell Biol 75, 606-616.

Received 24 November 1997; revised 17 November 1998; accepted 23 November 1998. 